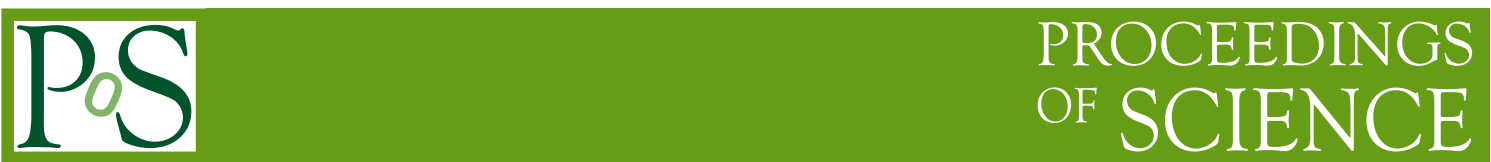

\title{
Evidence for high mass exclusive dijet production in the Do experiment
}

\author{
Zdenek Hubacek \\ Czech Technical University in Prague \\ E-mail: zdenek. hubacek@cern.ch \\ on behalf of the DO collaboration
}

Exclusive diffractive Higgs boson production is an interesting process which could be studied at the Large Hadron Collider. While the cross section for the Higgs boson production at the Fermilab Tevatron Collider is too low for this channel, it is important to check if the class of exclusive diffraction events exists. We present the evidence for the high mass exclusive dijet production in the D0 experiment [1].

XVIII International Workshop on Deep-Inelastic Scattering and Related Subjects April 19 -23, 2010

Convitto della Calza, Firenze, Italy 


\section{Introduction}

Hard diffractive processes are usually described by the exchange of a colorless object called Pomeron. In diffractive hadron hadron collisions, the hadrons will exchange the Pomeron and either one or both hadrons will not dissolve. The events are identified by either a presence of a large forward region of the detector devoid of any activity (rapidity gap) or by a tagging of the intact beam hadron(s). A subset of diffractive events is called exclusive when the whole Pomeron energy is used to produce the diffractive state, i.e there are no Pomeron remnants. Exclusive diffractive production (EDP) of the Higgs boson or any other new final state $X p p \rightarrow p+X+p$ has been recently proposed as a search channel at the LHC [2]. The cross section for the Higgs boson production is too low at the Tevatron $(0.2 \mathrm{fb}$ is predicted for a Higgs boson mass of $120 \mathrm{GeV})$, but it is important to check if this class of events exists in this kinematic region. The CDF Collaboration has recently confirmed the existence of EDP in several channels [3]. In this report, we present the evidence for the exclusive production of high dijet invariant mass events, i.e. a dijet event accompanied by large rapidity gaps on both sides of the calorimeter.
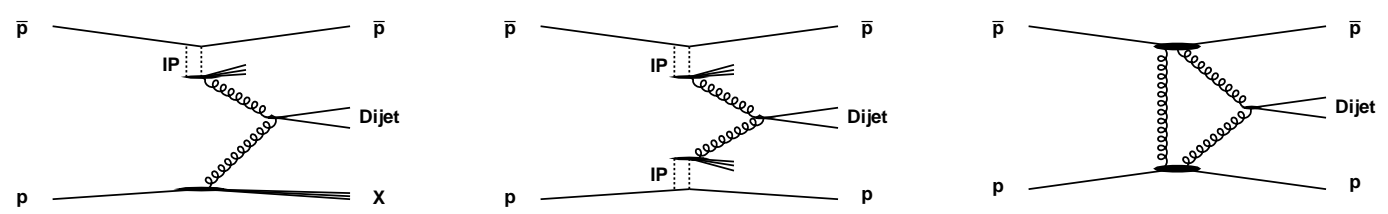

Figure 1: Classification of hard diffractive events. Single diffraction (left) where only one of the protons is diffracted, while the other breaks up. Inclusive double diffraction with two Pomeron remnants (middle) and exclusive diffraction (right) where both protons remain intact and only a dijet is produced in the central region.

\section{Data Selection and Separation}

The data used in this analysis were collected by the D0 detector between August 2002 and April 2006. The D0 detector is in detail described in [4], the most relevant component for this analysis is the calorimeter. The D0 uranium liquid argon calorimeter is divided into three separate cryostats - central, covering the pseudorapidity $|\eta|<1.1$ (where $\eta=-\ln \tan \frac{\theta}{2}, \theta$ is the polar angle), two endcap cryostats cover the regions $1.6<|\eta|<4.2$. The events are selected using a inclusive jet trigger which requires at least one jet above a transverse momentum $\left(p_{T}\right)$ threshold of $45 \mathrm{GeV}$. The offline event selection requires at least two jets to be reconstructed using an iterative D0 Run II midpoint cone algorithm [5] with a cone radius of $R=0.7$. High $p_{T}$ exclusive dijet events are expected to be produced more centrally than other jet production, therefore both jets in the analysis are required to be in the central detector with jet rapidities $|y|<0.8$ (where $y=0.5 \ln \left[E+p_{z}\right] /\left[E-p_{z}\right], E$ is the jet energy and $p_{z}$ is the jet momentum along the beam axis). The highest $p_{T}$ and second highest $p_{T}$ jets are required to have transverse momentum higher than $60 \mathrm{GeV}$ and $40 \mathrm{GeV}$ respectively. In order to suppress additional QCD radiation, the two jets are required to be back to back in the azimuthal angle $\phi$ with a separation $\Delta \phi>3.1 \mathrm{rad}$. Only dijet events with an invariant mass $M_{j j}>100 \mathrm{GeV}$ are selected. Additional event selection requirements include a cut on primary vertex $z$-coordinate to ensure a good reconstruction efficiency 
$\left(\left|\mathrm{PV}_{z}\right|<50 \mathrm{~cm}\right.$, at least 3 tracks associated with the primary vertex), a cosmic ray suppression cut on the missing transverse energy in an event (missing transverse energy has to be less than $70 \%$ of the leading jet transverse momentum) and a cut on the instantaneous luminosity which limits the contamination of rapidity gaps due to the energy from additional interactions in the same bunch crossing (instantaneous luminosity is restricted to $[5-100] \times 10^{30} \mathrm{~cm}^{-2} \mathrm{~s}^{-1}$ ). Due to the prescales to avoid the saturation of the data acquisition system, the final luminosity of the sample is about $30 \mathrm{pb}^{-1}$.

The Monte Carlo events are required to satisfy the same selection criteria as in data. The following samples are used in the analysis. The nondiffractive (NDF) events are generated with PYTHIA [6], single diffractive (SD) and inclusive double diffractive (IDP) backgrounds are determined using the POMWIG [7] and FPMC [8] generators respectively. The signal EDP events were generated using FPMC. These events are processed through a GEANT-based simulation of the D0 detector and use the same reconstruction code as the data. The MC events are reweighted for the trigger inefficiency observed in the leading jet $p_{T}$ range between 60 and $100 \mathrm{GeV}$. Random data events are overlaid over the generated MC events, the MC events are reweighted in addition to obtain the same instantaneous profile as in the data to obtain the same noises and energies in the forward region of the calorimeter. The sum of NDF, SD and IDP, each one weighted by their cross section, is normalized to data. The EDP contribution is negligible at this stage. Figure 2 shows the good agreement between the MC simulation and data after this rescaling. By varying the cut on the leading jet $p_{T}$, the uncertainty on the normalization was estimated to be $5 \%$.

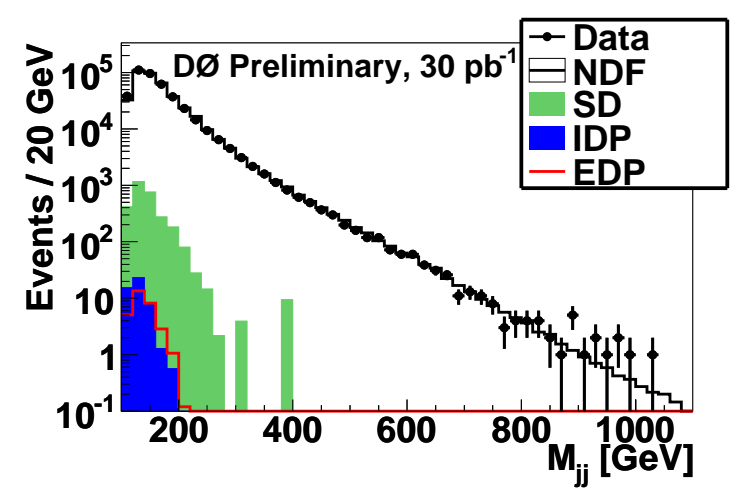

Figure 2: Dijet invariant mass distribution in MC and data.

A discrimination variable is chosen to separate the EDP events and the background (NDF, SD and IDP). Since large rapidity gaps are expected on both sides of the exclusively produced dijet system, the variable is based on the sum of transverse energies in the forward regions of the calorimeter. The very forward region $|\eta|>3.0$ is able to discriminate between the nondiffractive and diffractive events since this region contains the proton remnants. The intermediate forward region $2.0<|\eta|<3.0$ is used to identify EDP events since they show larger rapidity gaps. The discrimination variable $\Delta$ defined in Eq. 2.1 is formed by summing the transverse energies of cells in the considered region. Noisy cells in the forward region with an occupancy larger than 5 standard deviations from the average, are excluded in the sum. In addition, the cell response in the MC was adjusted to data using a MC-to-data correction factor for each cell. Figure 3 shows the normalized 
distribution of the $\Delta$ for all MC samples (left) and comparison of $\Delta$ between data and MC (NDF, SD and IDP) normalized to their leading order cross section (right). Good agreement is observed except at high values of $\Delta$ where EDP dominates.

$$
\Delta=\frac{1}{2} \exp \left(-\sum_{2.0<|\eta| \leq 3.0} E_{T}\right)+\frac{1}{2} \exp \left(-\sum_{3.0<|\eta|<4.2} E_{T}\right)
$$
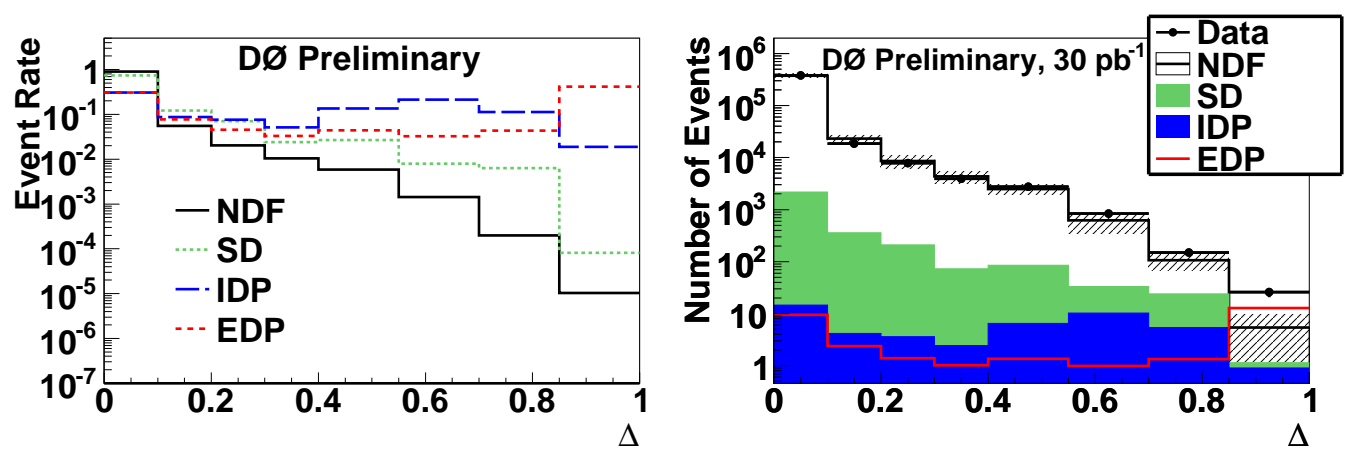

Figure 3: $\Delta$ discriminant normalized to unity for all MC samples (left) and $\Delta$ distribution for data and MC (right). A good agreement is observed except at high values of $\Delta$ where EDP dominates. The hatched band indicates the total uncertainty on the background.

The significance of the excess at high $\Delta$ is determined using a modified frequentist approach [9]. It is obtained by fitting signal and background hypotheses to pseudodata samples containing only background. The pseudoexperiments include the variations of the fluctuations over each systematic uncertainty (the dominant uncertainties come from the cell calibration factors, where a variation of three standard deviations from their central value changes the background by $25 \%$ for $\Delta \geq 0.85$ and jet energy scale, which changes the background by $12 \%$. The observed significance corresponds to the fraction of outcomes of pseudoexperiments that yield the EDP cross section at least as large as measured in data. The probability for the observed excess to be explained by a fluctuation of the background is $2 \times 10^{-5}$ which corresponds to an excess of 4.1 standard deviations.

\section{Conclusions}

We have presented evidence at the 4.1 standard deviations level for the exclusive high mass dijet production. The separation variable $\Delta$ is formed from a sum of calorimeter cell energies to discriminate between nondiffractive, inclusive and exclusive diffractive events. The dijet invariant mass distribution of events with $\Delta>0.85$ dominated by the exclusive events is shown in Fig. 4. Figure 5 shows a comparison of an exclusive diffraction event with large rapidity gaps and a standard nondiffractive event. Exclusive diffraction event signatures can play an important role in future studies at the Tevatron and LHC.

The author is supported by the grant MSM6840770039 of the Czech Ministry of Education.

\section{References}

[1] V. M. Abazov et al., (D0 Collaboration), Conference note 6042-CONF. 


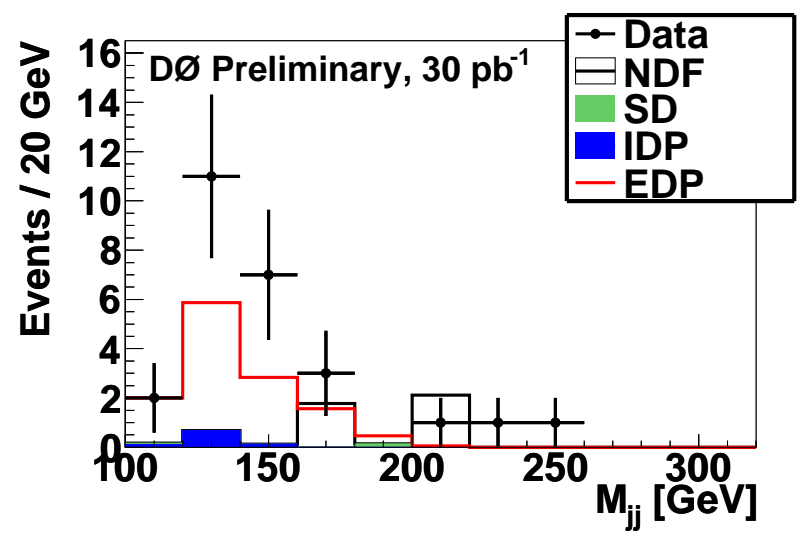

Figure 4: Dijet invariant mass distribution after applying the cut on $\Delta \geq 0.85$.
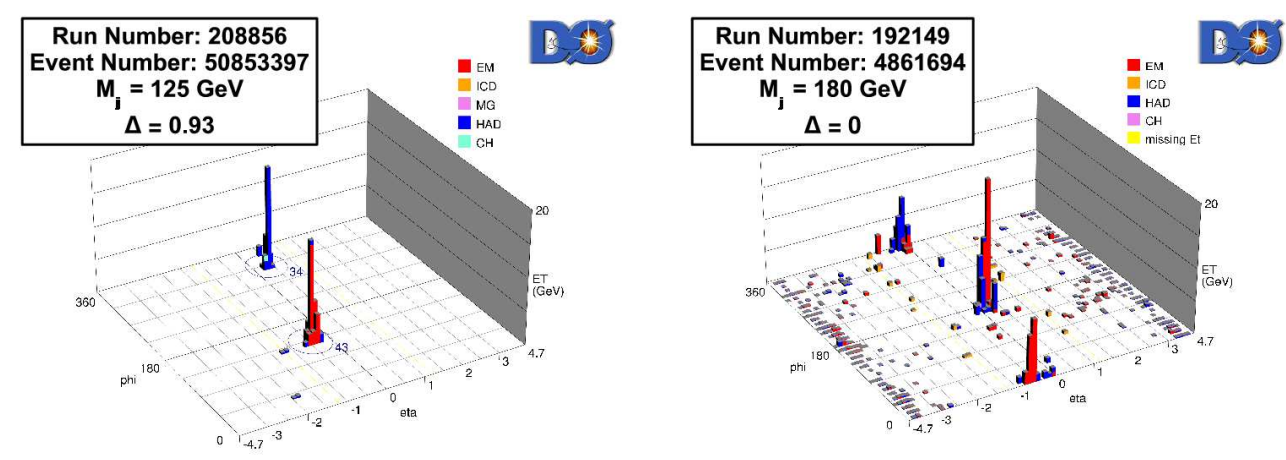

Figure 5: D0 event displays for an exclusive diffractive event (left) and a background event (right). The difference between them is the amount of additional energy depositions in the forward region.

[2] M. Boonekamp, R. B. Peschanski and C. Royon, Phys. Lett. B 598, 243 (2004); C. Royon, (RP220 Collaboration), arXiv:0706.1796 [physics.ins-det]; M. G. Albrow et al., (FP420 R\& D Collaboration), arXiv:0806.0302 [hep-ex].

[3] T. Aaltonen et al., (CDF Collaboration), Phys. Rev. D 77, 052004 (2008); T. Aaltonen et al., (CDF Collaboration), Phys. Rev. Lett. 99, 242002 (2007); T. Aaltonen et al., (CDF Collaboration), Phys. Rev. Lett. 102, 222002 (2009); T. Aaltonen et al., (CDF Collaboration), Phys. Rev. Lett. 102, 242001 (2009); A. Abulencia et al., (CDF Collaboration), Phys. Rev. Lett. 98, 112001 (2007).

[4] V. M. Abazov et al., (D0 Collaboration), Nucl. Instrum. Methods Phys. Res. A 565, 463 (2006).

[5] G. Blazey et al. in Proceedings of the Workshop: QCD andWeak Boson Physics in Run II, edited by U. Baur, R.K. Ellis, and D. Zeppenfeld, Fermilab-Pub-00/297 (2000).

[6] T. Sjöstrand et al., Comput. Phys. Commun. 135, 238 (2001).

[7] B. E. Cox and J. R. Forshaw, Comput. Phys. Commun. 144, 104 (2002) [arXiv:hep-ph/0010303].

[8] M. Boonekamp, V. Juranek, O. Kepka, C. Royon, Proceedings of the Workshop of the Implications of HERA for LHC physics, DESY-Proc-2009-02.

[9] W. Fisher, FERMILAB-TM-2386-E (2006). 